\title{
Qualité de l'eau et maladies hydriques dans la commune de Toffo (Bénin, Afrique de l'ouest)
}

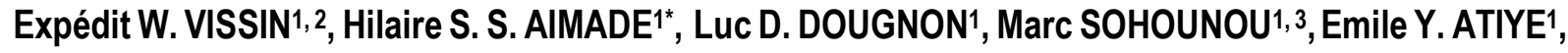 \\ Gervais A. A. ATCHADE ${ }^{1,2}$ \\ 1Département de Géographie et Aménagement du Territoire (Université d'Abomey-Calavi). \\ 2Laboratoire Pierre Pagney "Climat, Eau, Écosystèmes et Développement" (LACEEDE), Université d'Abomey-Calavi \\ (Bénin). \\ ${ }^{3}$ Laboratoire de Recherche en Biochimie et Toxicologie de l'Environnement (LaRBiTE), Département de Biochimie et de \\ Biologie Cellulaire, Faculté des Sciences et Techniques (FAST), Université d'Abomey-Calavi (UAC), BP 2899 Abomey- \\ Calavi, Bénin \\ *Auteur correspondant E-mail : aimadehilaire@yahoo.com \\ borismarcs@gmail.com, dosg09@yahoo.fr, ativeemile@gmail.com, exlaure@gmail.com
}

Original submitted in on $15^{\text {th }}$ August 2016. Published online at www.m.elewa.org on $31^{\text {st }}$ October 2016 http://dx.doi.org/10.4314/jab.v106i1.10

\section{RESUME}

Objectif : L'objectif de cette étude est de contribuer à une amélioration de la qualité de l'eau des populations dans la Commune de Toffo.

Méthodologie et résultats : La méthodologique s'articule autour de la collecte des données, du traitement des données et l'analyse des résultats qui est faite à l'aide du modèle FPEIR. A partir des prélèvements d'échantillon d'eau effectué, au niveau d'un (01) puits moderne, d'un (01) forage et d'une (01) rivière, des analyses Bactériologique et Physico-chimique ont été effectuées. L'analyse des échantillons d'eau prélevés permet d'obtenir des paramètres (couleur : puits $=8$; rivière $=19$ et $\mathrm{PH}$ : puits $=5,6$; forage $=6,0$ ) qui sont supérieures à la norme (couleur $=15$ et $6,5<\mathrm{pH}<8,5$ ). Ces échantillons d'eau sont également très contaminés par des germes bactériologiques (coliformes fécaux, coliformes totaux, et streptocoques fécaux).

Conclusion et applications : L'eau est soumise à une pollution environnementale dans la commune de Toffo. Cette pollution d'origine anthropique est liée à des eaux usées, ordures ménagères et de défécation.

Mots clés : Toffo, pollution de l'eau, qualité de l'eau, environnement

\begin{abstract}
Objective: The objective of this study is to contribute to improving the water quality of the people in the Municipality of Toffo.

Methods and Results: The methodology is based on data collection, data processing and analysis of results that is made using the PEIR. From the water sample taken samples, at a (01) modern wells, one (01) a borehole and (01) river, where Bacteriological and Physico-chemical analyzes were performed. Analysis of water samples provided the parameters (color: well $=8$; river $=19$ and $\mathrm{PH}$ : Well $=5.6$; Bore hole $=6.0$ ) which
\end{abstract}


are superior to the standard (color $=15$ and $6.5<\mathrm{pH}<8.5$ ). These water samples were also highly contaminated with bacteria germs (fecal coliforms, total coliforms and faecal streptococci).

Conclusion and Applications: Water is subjected to environmental pollution in the town of Toffo. This anthropogenic pollution is related to sewage, garbage and defecation.

Keywords: Toffo, water pollution, water quality, environment

\section{INTRODUCTION}

La vie sur terre est possible grâce à l'existence de certaines ressources vitales dont l'eau, denrée de grande importance pour les êtres vivants (Housssou, 2010). Sans elle, il n'y aurait pas de vie sur terre. Les plantes et les animaux en ont besoin pour satisfaire leurs besoins quotidiens (Blalogoé, 2002). Elle joue un rôle déterminant dans la vie des hommes, des animaux et des plantes (Avocetien, 2012). Selon l'OMS (2014), qu'environ $2,5 \%$ de l'eau présente sur terre est de l'eau douce, en bonne partie inaccessible car elle est la plus importante des ressources naturelles qui rendent la vie possible, soutient les écosystèmes et les entreprises de l'Homme. L'eau est donc à la fois une ressource stratégique et l'élément de base fondamental nécessaire à une économie saine (Odoulami, 1999) cité par Ayéna (2009). De ces multiples usages qu'on peut donc faire de l'eau, celui relatif à l'alimentation et au développement des hommes, est

\section{MATERIELS ET METHODES}

Zone d'étude : La Commune de Toffo est situé entre $6^{\circ}$ $43^{\prime} 06^{\prime \prime}$ et $6^{\circ} 57^{\prime} 48^{\prime \prime}$ de latitude nord et $2^{\circ} 02^{\prime} 09^{\prime \prime}$ et $2^{\circ}$ $20^{\prime} 56^{\prime \prime}$ de longitude est. Couvrant une superficie de 492 $\mathrm{km}^{2}$, elle est limitée au Nord par la Commune de Zogbodomey dans le département du Zou, à l'Est par la Commune de Zè, au Sud par la Commune d'Allada et à l'Ouest par le fleuve Couffo servant de frontière naturelle avec la Commune de Lalo dans le département du Couffo (Figure 1)

Données : Pour bien mener cette étude, des données ont été collectées. II s'agit: primordial (Tandjè, 2009). Pour (Tchaba, 2012) la qualité de l'eau utilisée pour satisfaire ces différents besoins varie d'un usage à un autre. Pour (Babadjidé, 2011) la qualité de l'eau de distribution, est l'enjeu d'une compétition économique, que se livrent à de grands groupes industriels, pour ce que certains nomment «l'or bleu ».Pour (Babadjidé, 2001), l'un des éléments qui affecte la santé est l'eau polluée, de mauvaise qualité qui constitue l'une des causes de maladies altérant la santé de l'homme. II continu en disant que l'eau constitue une source de vie, mais elle est également source de maladie de part sa dégradation et sa mauvaise gestion. Les offres de service hydraulique par la DGEau sont loin de couvrir les besoins en eau des populations, qui trouvent satisfaction à leur besoin en eau par l'usage des sources habituelles environnantes qui sont les eaux de surface et de puits.

- données démographiques (effectifs de la population et le nombre de ménage) (INSAE, 2013) ;

- données climatologiques (hauteurs de pluies et températures) à l'échelle mensuelles et annuelles) sur la période allant de 1977 à 2012 de la station de Toffo ;

- données cartographiques (cartes de situation géographique et hydrologique) ;

- données épidémiologiques de 2008 à 2012 relatives aux différentes pathologies tirées du registre du centre de santé de la Commune et les données relatives aux normes de potabilité de l'eau fixées par l'OMS (2008) et la direction générale de l'eau du Bénin. 


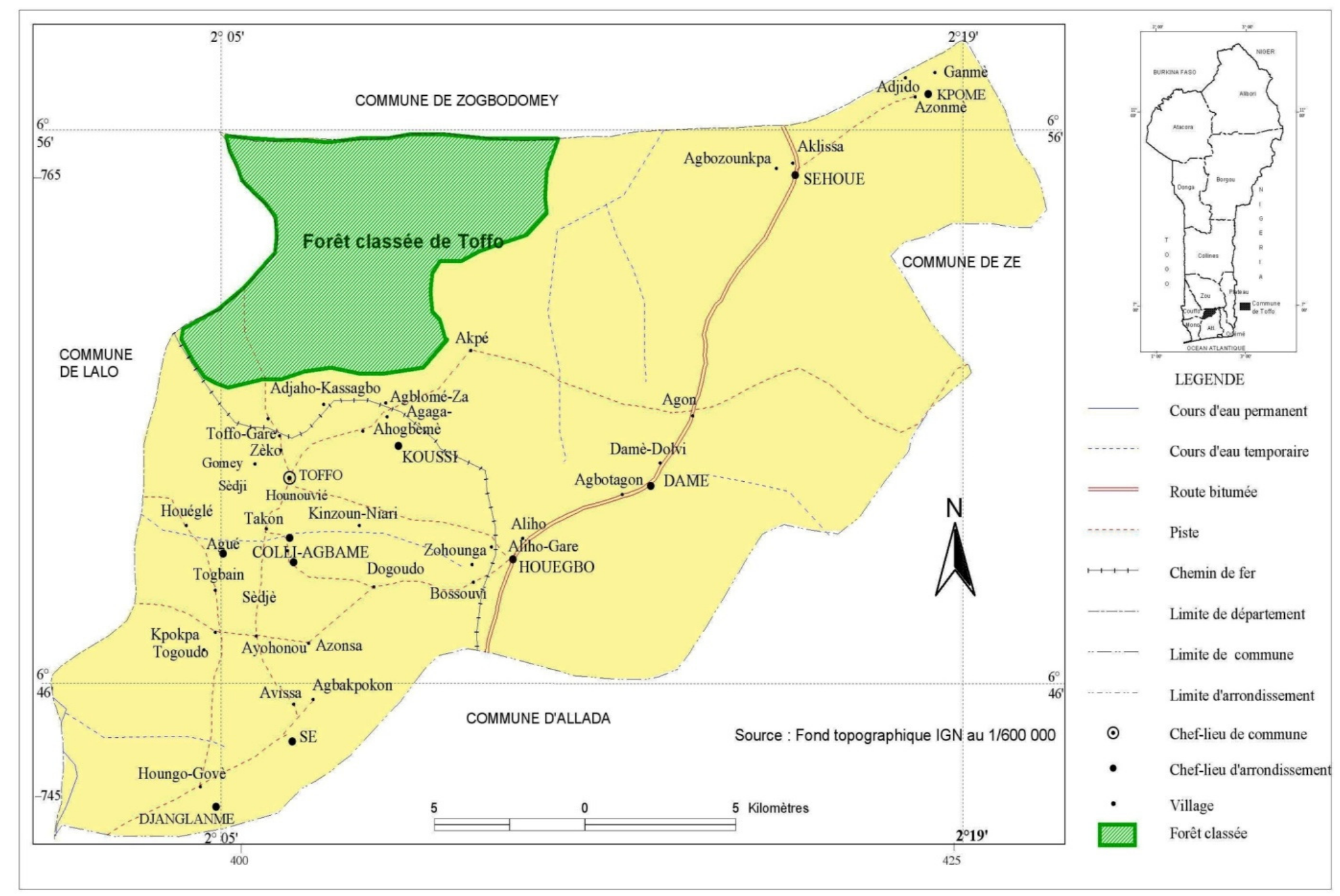

Figure 1 : Situation géographique de la Commune de Toffo 
Échantillonnage: L'échantillon aléatoire simple au niveau de chacun des 05 arrondissements choisis a été fait pour déterminer le nombre de ménages visités dans la Commune. La taille de l'échantillon par arrondissement a été déterminée à partir de la méthode de Schwartz (1995). La formule s'écrit: $\mathrm{Na}=\mathrm{Za}^{2} \mathrm{P} Q / \mathrm{i}^{2}$ avec $\mathrm{Na}=$ taille de l'échantillon par arrondissement ; $Z a=$ écart fixé à 1,96 correspondant à un degré de confiance de $95 \%$ $P=n / N$ avec $p=$ proportion des ménages des cinq (05) arrondissements (n) par rapport au nombre de ménage dans la Commune de Toffo (N). Dans le cadre de cette étude, $p$ s'obtient par la méthode suivante :
$P=4697 / 17054=0,27$ avec $P=27 \% ; i=$ marge d'erreur qui est égale à $5 \%$

$Q=1-p$ avec $Q=1-0,27=0,73$

Dans le cadre de cette étude, la taille réelle de l'échantillon est de : $\mathrm{Na}=(1,96)^{2} \times 0,27 \times 0,73 /(0,05)^{2}=$ 302

Analyse des données: Le cadre conceptuel est un schéma cohérant comme l'indique la figure 2, mettant en relation les concepts clés du sujet, il représente la «base» du travail de recherche.

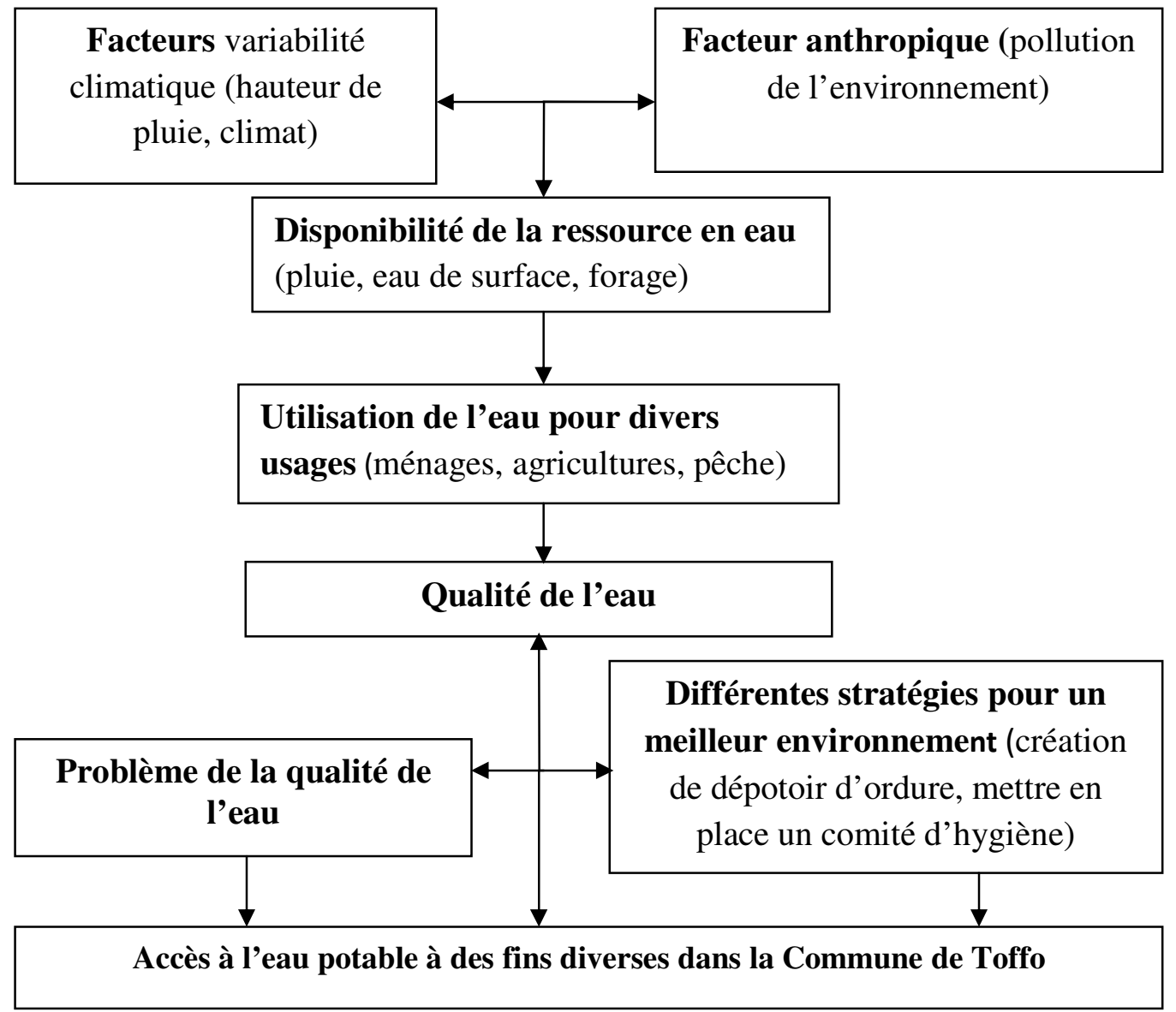

Figure 2: Schéma conceptuel relatif à l'accès à l'eau potable à des fins diverses dans la Commune de Toffo Source : Sokadjo, 2014

L'analyse des résultats a été faite grâce au modèle PEIR qui se définit comme Forces- Pression- État- ImpactRéponses. Ce modèle a permis d'évaluer les impacts de l'environnement sur la qualité de l'eau. La figure 5 est utilisée pour connaitre les causes de la pression des impacts sur le milieu. Le modèle a permis d'établir les liens entre la qualité de l'eau et l'état de l'environnement social ou physique. 
RESULTATS ET DISCUSSIONS

Sources d'approvisionnement : Les populations de la Commune de Toffo disposent de plusieurs sources d'approvisionnement en eau. II s'agit des eaux de pluie, de forages, de puits, de marigot et même de rivière.

- $\quad$ Eaux de pluie : Pendant la saison des pluies, les populations des arrondissements d'Agué, Coussi, Houègbo, Kpomè et Sèhouè enquêtées utilisent les eaux des toits de maisons à ciel couvertes de tôles ondulées recueillies dans des bassines, des jarres, des tonneaux, des citernes et même directement pour leurs besoins domestiques. Cette eau peut être source de maladie hydrique des populations car les toits sont souvent construits en matériaux précaires et tout s'y dépose. On note que $38,12 \%$ des populations interrogées utilisent l'eau de citerne en saison pluvieuse. Les photos 1 et 2 de la planche 1 montrent une manière d'approvisionnement en eau de citerne et de pluie pendant qu'il pleut par les populations.
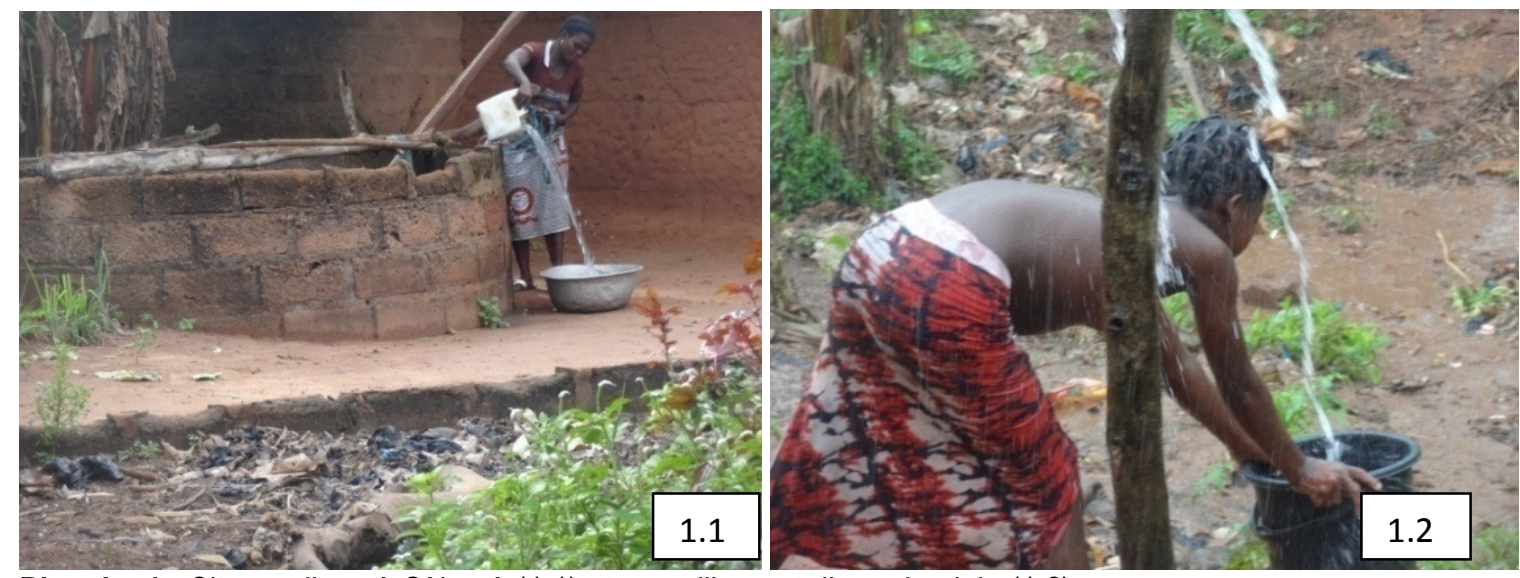

Planche 1 : Citerne d'eau à Sèhouè (1.1) et recueillement d'eau de pluie (1.2)

Prise de vue : Sokadjo, Septembre 2014

L'approvisionnement en eaux à cette source a des implications sur la santé des populations. Les enquêtes ont montré que par manque de moyens et aussi de la couleur de l'eau que fournissent certaines bonnes fontaines, $50 \%$ des populations font recours à ces sources d'eau qui se trouvent dans un environnement insalubre ce qui a des impacts direct sur la qualité de l'eau et donc sur la santé des populations.
- $\quad$ Eau de rivière : Sur les cinq arrondissements enquêtés dans la commune de Toffo, deux entre eux utilisent les eaux de rivière que sont Agué et Kpomè. A Agué, comme à Kpomè, $13 \%$ des populations utilisent ces sources pour raison de proximité et surtout que l'AEV de ces deux arrondissements est en panne. La planche 2 montre les photos des rivières d'approvisionnement en eau dans l'arrondissement d'Agué.
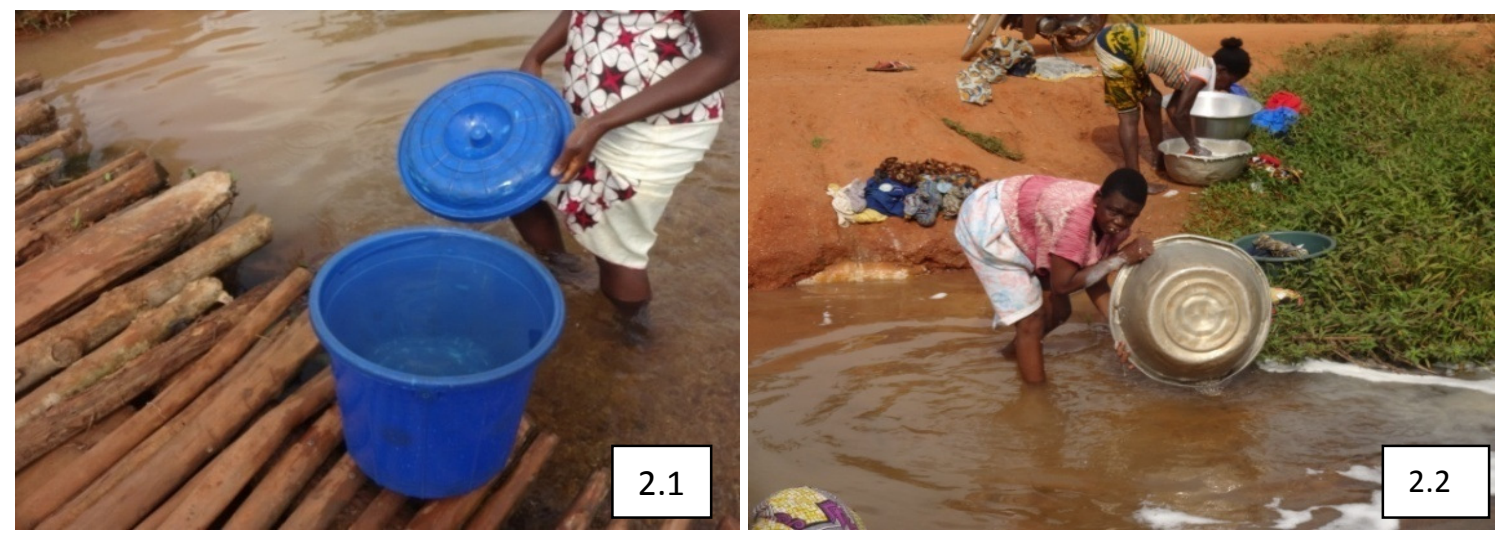

Planche 2 : Rivière à Agué Pise de vue : Sokadjo, Septembre 2014 
La planche 2 montre que l'eau de rivière est une source d'approvisionnement d'eau pour l'utilisation à des fin domestique et en même temps source de lessive et de baignade pour d'autre.

Eaux de puits : Les puits elles sont de deux sortent : les puits traditionnels et les puits à grand diamètre (moderne).

- Puits traditionnels : Ils existent dans quelques-uns des arrondissements de la commune. $17 \%$ des populations utilisent cette source et elles ne prennent même pas la peine de la protéger et ignorent les risques de consommation du à un environnement insalubre. L'eau de puits est souvent utilisée pour raison de proximité, de coût et surtout de la qualité d'eau que fourni certaine AEV comme le cas de I'AEV de Sèhouè ou le nouveau château fournit une eau salée donnant une odeur nauséabonde et les populations l'ont abandonné au profit des eaux de puits et de pluie.
- Puits à grand diamètre (moderne): Malgré les multiples efforts consentis par l'État béninois, les organismes étrangers et les ONG à travers le service de l'hydraulique, les puits modernes sont très insuffisants pour satisfaire les besoins en eau des populations. A la différence des puits traditionnels, les puits modernes sont entièrement crépis et une margelle limite la pollution de l'eau. Ces puits sont plus présents dans l'arrondissement de Coussi. L'AEV de cet arrondissement étant en panne depuis 3 ans, $11 \%$ des populations interrogées dans cet arrondissement n'utilisent que l'eau de ce puits. Elle est protégée et l'eau est vendue aux populations au prix de 25 FCFAle bidon de $50 \mathrm{~L}$. Les sous collectés sont destinés à l'entretien du puits. La planche 3 montre les puits d'approvisionnement en eau à Coussi.
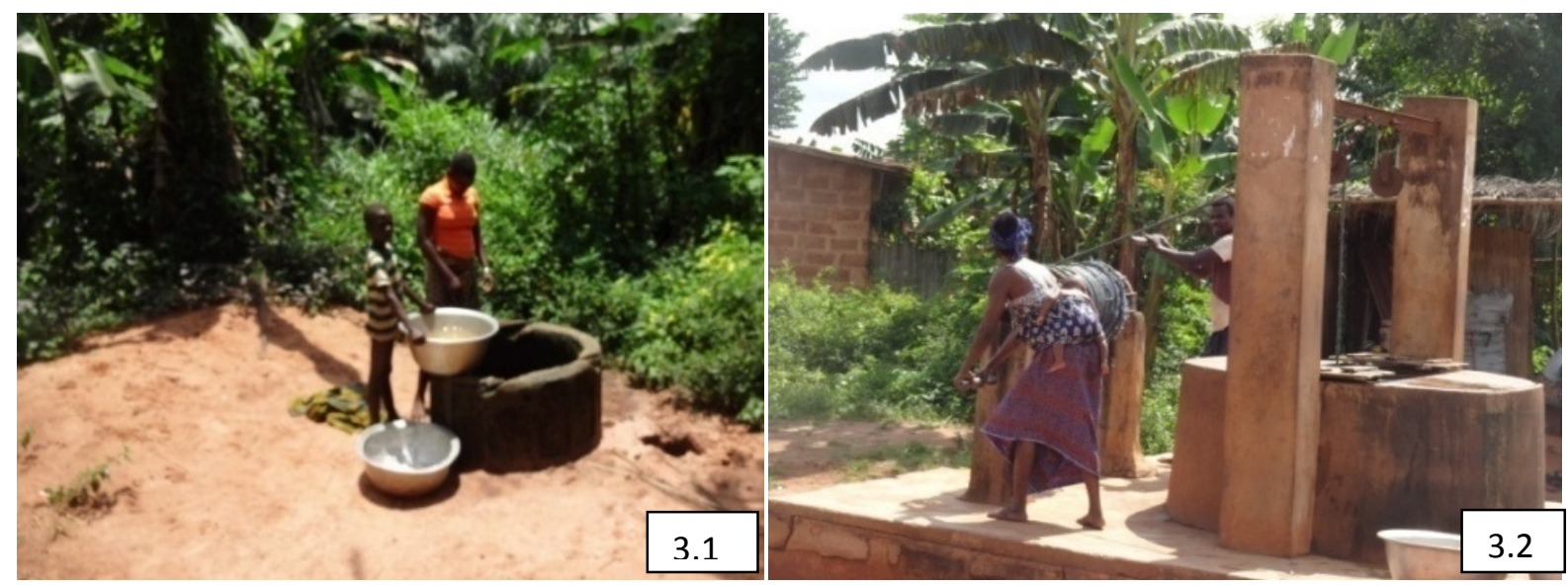

Planche 3: Puits traditionnel à Coussi (3.1) et puits moderne à Coussi (3.2)

Pise de vue : Sokadjo, Septembre 2014

La planche 3 montre les puits d'approvisionnement en eau des populations. Les eaux de ces sources ne sont pas totalement exemptes de pollutions. Elles peuvent d'une part être contaminées par la technique de puisage, la proximité des feuillages ou d'autres sources de pollution.

Eau de bornes fontaines : L'eau de la borne fontaine est la source qui fournit l'eau potable et vendue à la population à la place de l'eau de la SONEB. Pour faute d'extension ou en cas du dysfonctionnement de celle-ci ou quand elle fournit une eau de mauvaise qualité, les populations sont obligées d'abandonner ces sources au profit des sources alternatives. La gestion de ces BF est confiée à un fermier qui gère l'entretien et leur réparation. La planche 4 montre quelques bornes fontaines. II est à noter que $20,77 \%$ des personnes interrogées utilisent l'eau de cette source. 

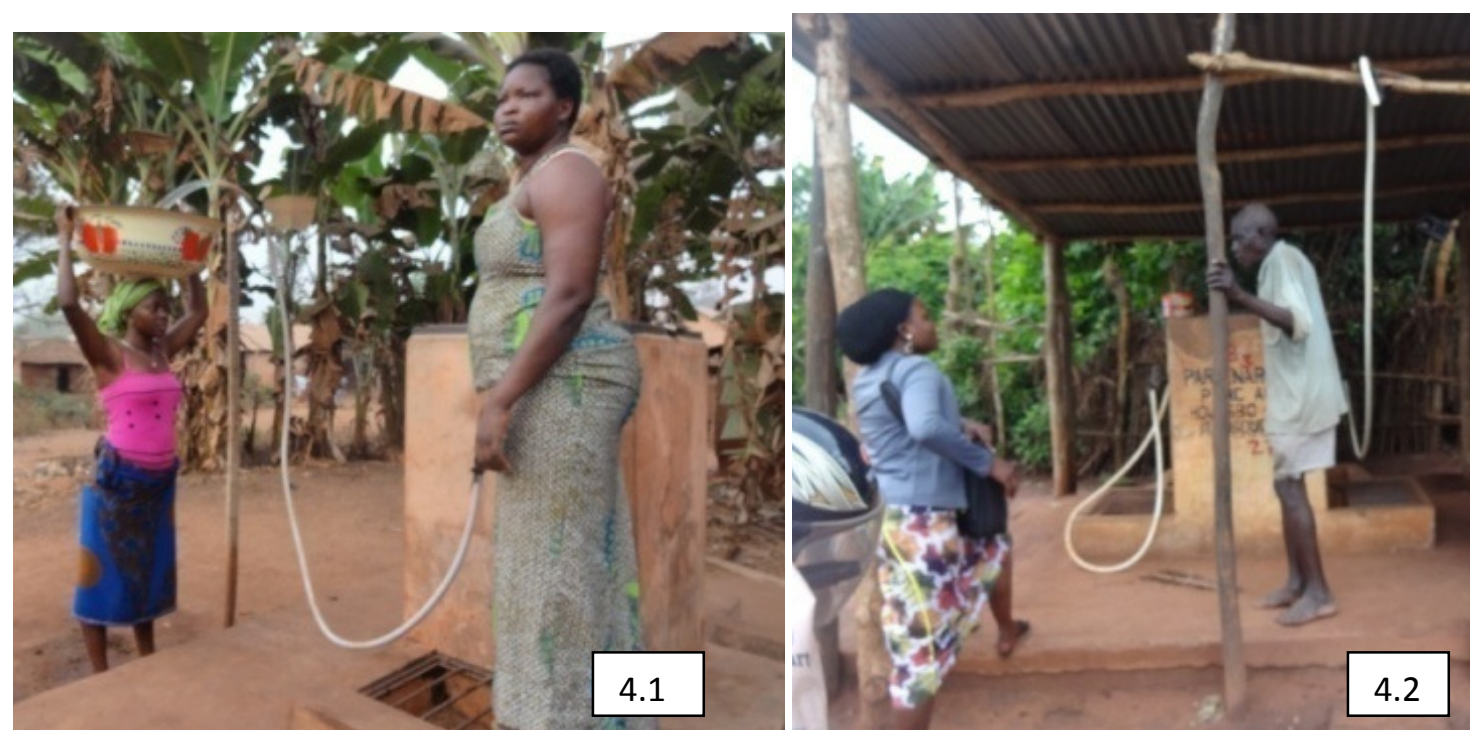

Planche 4 : Borne fontaine à Sèhouè (4.1) et à Houègbo (4.2)

Prise de vue : Capko, Septembre 2014

La planche 4 montre l'état dans lequel se trouvent les bornes fontaines $(\mathrm{BF})$. Ces $\mathrm{BF}$ ne sont pas entretenues comme il le faut par les gestionnaires et les usagers de ces points d'eau. Ainsi donc, même si l'eau qui sort du robinet est propre, l'état des lieux peut la contaminer dans les minutes qui suivent. Dans ce cas, ce n'est plus l'eau du robinet qui est d'une qualité douteuse mais plutôt l'entourage qui la rend douteuse. On n'observe pas d'affluence à ces bornes fontaines parce qu'on est en pleine saison des pluies et les populations ont abandonné ces sources au profit de l'eau de pluie qui est gratuite pendant que celle de la borne fontaine est vendue.
Affections liées à l'eau dans la Commune de Toffo: Dans la Commune de Toffo, plusieurs maladies sont enregistrées telles que le paludisme, les affections cardiaux vasculaires, etc. Le paludisme, il est le premier cas de maladie enregistré dans cette Commune. Ensuite, viennent les infections respiratoires aigües. Les maladies hydriques, sont les troisièmes (3eme) cas de maladies enregistrées dans la Commune. C'est ce qu'indique la figure 3 (Palu= Paludisme; IRA= Infection respiratoire Aigües ; Mala= Maladies Hydriques; AVC= affections cardiaux vasculaires ; Affec $=$ affections dermatologiques)

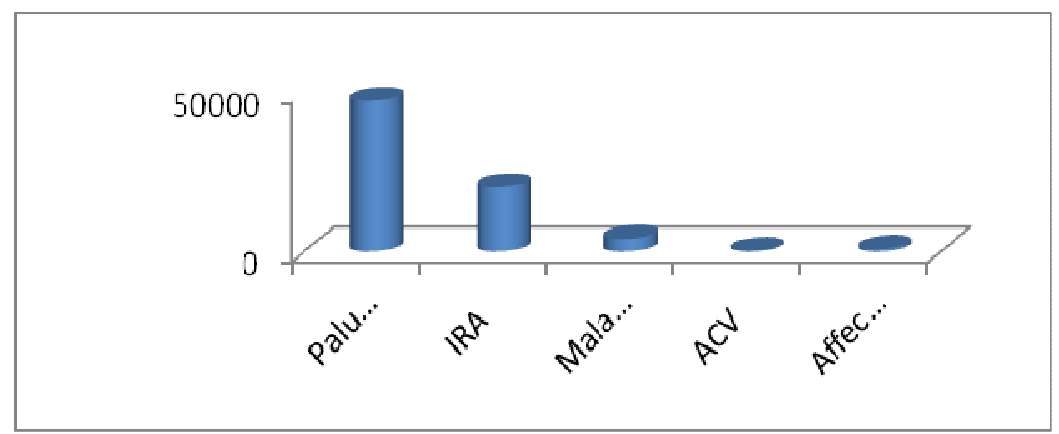

Figure 3 : Cas des maladies enregistrées dans la commune de Toffo

Source : Donnée CSC/Toffo, Septembre 2014

De l'analyse de la figure 3 , on constate que le paludisme est la maladie qui sévit le plus dans la commune. Les infections respiratoires aiguës, les affections dermatologiques et les affections cardiaux vasculaires sont pratiquement négligeables dans la Commune. Enfin, les maladies hydriques (4020 cas enregistrés) sont de plusieurs ordres et menacent dangereusement la santé des populations. 
Principales maladies liées à l'eau dans la commune : Les maladies liées à l'eau de boisson résultent d'un contact direct avec l'eau ou de la nourriture contaminée par des déchets d'origine humaine, animale ou chimique. L'OMS 2015 comptabilise 4milliards d'infections gastrointestinales dues à des bactéries, virus ou parasites par an. Elles ont mené à mort 1,8 millions de personnes en 2002. Ces maladies sont particulièrement liées à la pauvreté. Les enfants de moins de 5 ans sont les plus touchés. Au nombre des maladies hydriques enregistrées dans la Commune de Toffo, on peut noter la bilharziose, la diarrhée, la dracunculose et le choléra.

- Diarrhée: Elle est causée par divers microorganismes parmi lesquels: des virus, des bactéries, et des protozoaires. La diarrhée fait perdre à des personnes à la fois de l'eau et des électrolytes; ce qui entraine une déshydratation et dans certains cas, la mort. Cette maladie est contractée en buvant de l'eau contaminée par des larves responsables de cette maladie. La figure 4 montre l'évolution des cas de diarrhée enregistrés entre 2009 - 2013 dans la Commune de Toffo.

- Bilharziose : Elle est due à des vers plats appelés schistosomes vivant dans les vaisseaux sanguins. Les êtres humains contractent cette maladie au cours des contacts avec une eau douce contaminée dans laquelle se trouvent les mollusques qui sont les hôtes intermédiaires à l'origine des larves infectantes et sans lesquelles les parasites ne peuvent effectuer leur cycle. La figure 5 présente l'évolution des cas enregistrés entre $2009-2013$.

- Dracunculose : Type de gastropodes d'eau douce, dans l'eau (ou ils peuvent survivre 48 heures) et dans leurs hôtes humains. Ils entrent dans le corps de ceux qui se baignent dans de l'eau contaminée. Ils causent des infections pouvant endommager le foie, les intestins, les poumons et les vessies. La figure 6 présente l'évolution des cas enregistrés entre 2009 - 2013.

Tableau 1 : Évolution de quelques cas de maladies dans la Commune de Toffo

\begin{tabular}{|l|l|l|l|}
\hline Année & $\begin{array}{l}\text { Diarrhée } \\
\text { (Nombre des cas) }\end{array}$ & $\begin{array}{l}\text { Bilharziose } \\
\text { (Nombre des cas) }\end{array}$ & $\begin{array}{l}\text { Dracunculose } \\
\text { (Nombre des cas) }\end{array}$ \\
\hline 2009 & 1100 & 175 & 800 \\
\hline 2010 & 1500 & 650 & 20 \\
\hline 2011 & 800 & 300 & 5 \\
\hline 2012 & 1000 & 320 & 0 \\
\hline 2013 & 1000 & 200 & 0 \\
\hline
\end{tabular}

Source : Donné CSC/Toffo, Septembre 2014

L'analyse du tableau présente le nombre de cas de diarrhée enregistré dans l'espace de 05 ans est faible. Ce n'est qu'en 2010 que la Commune de Toffo a enregistré plus de cas de diarrhée. La diarrhée est la deuxième cause de mortalité chez les enfants de moins de cinq ans. Elle peut durer plusieurs jours, déshydratant l'organisme et le privant des sels minéraux nécessaires pour la survie. Elle est en général le symptôme d'une infection intestinale pouvant être causée par divers microorganismes, bactéries, virus ou parasites. L'infection se transmet par le biais de l'eau ou d'aliments contaminés, ou d'une personne à l'autre en cas d'hygiène insuffisante. Le tableau montre qu'en 2010 le nombre de cas de bilharziose enregistré est important $(46 \%$ de cas enregistrés). La bilharziose touche souvent les zones rurales. A Toffo, ce cas de maladie est plus enregistré dans l'arrondissement d'Ague plus précisément dans le village de Takon ou $90 \%$ des populations sont en contact avec l'eau de rivière. Dans l'espace de 5 ans, ce cas d'affection est plus enregistré en 2009 (806 cas enregistrés). Elle ne se contracte que par consommation d'eau contenant des cyclopes qui sont les hôtes intermédiaires du parasite. Environ un an après l'ingestion, le ver, parvenu à maturité, commence à migrer à l'intérieur du corps du sujet infecté. Dans les pays d'endémie, la maladie se caractérise par une réapparition annuelle au moment de la saison agricole, les cultivateurs étant particulièrement touchés. II n'existe aucun médicament contre cette maladie. 


\section{CONCLUSION}

La Commune de Toffo comme est confrontée au problème de la qualité de l'eau. Elle est caractérisée par un environnement pollué. En effet, les populations de la Commune, de par leurs activités (économiques et ménagère), qui produisent des déchets. Sa mauvaise gestion de a un impact négatif sur la qualité de l'eau de consommation des populations de cette commune. Plusieurs affections menacent dans le milieu de vie. Au nombre de ces affections, le paludisme, les infections respiratoires aigues, les maladies hydriques sont les plus dominantes. Ces maladies sont imputables à la modification de l'environnement, à la mauvaise condition d'hygiène et d'assainissement et aux comportements des populations. Cette situation se caractérise par l'absence d'installation des ouvrages d'assainissement, la consommation d'eau insalubre et l'exposition aux polluants organiques. Quant aux maladies hydriques, elles sont dues à la consommation d'une eau de

\section{REFERENCES BIBLIOGRAPHIQUES}

Avocetien A.C., 2012. Conservation de l'eau de puits dans les maisons dans la commune de SOAVA : cas de SO-TCHANHOUE et de SOZOUNKO Mémoire de licence professionnelle EPAC/UAC, $50 \mathrm{p}$.

Ayéna G. M., 2009. Problématique de l'eau potable dans l'arrondissement de lissazounmè (Commune d'Agbangnizou).Mémoire de maitrise

FLASH/UAC, $81 \mathrm{p}$.

Babadjidé C. H., 2011. Pollution, ses conséquences, ses causes et ses incidences sur la santé humaine dans le bassin du fleuve Mono au Bénin Thèse de doctorat, $222 \mathrm{p}$.

Balogoe C. P., 2002. Eau et la santé publique en milieu de climat de transition: Étude de cas de la Commune de Glazoué. Mémoire de maîtrise FLASH/UAC, $114 \mathrm{p}$. mauvaise qualité ; or tout homme quelles que soient ses conditions de vie ou ses ressources, a le droit de boire une eau saine. II ne suffit pas d'avoir des ressources en eau en quantité suffisante; il faut également que la qualité de celle-ci (eau) soit satisfaisante pour les différents usages domestiques. Les résultats physicochimiques et bactériologiques obtenus au cours de l'étude ont révélé la présence de germes pathogènes d'origine fécale et animale dans des eaux. Les facteurs qui expliquent la pollution bactériologique de ces eaux sont liés au manque d'hygiène (absence de système de gestion des eaux usées), mais surtout au comportement des populations. Ces résultats montrent que les populations de la Commune de Toffo consomment souvent l'eau sans avoir la moindre idée de sa qualité. $\mathrm{Ce}$ qui traduit l'ignorance de la sensibilisation des populations sur les risques liés à la consommation d'une eau polluée.

Houssou C. J. L., 2010. Gestion de l'eau au Bénin et ses impacts environnementaux: Cas de l'arrondissement de Houin dans la Commune de Lokossa Mémoire de maitrise professionnelle FLASH/UAC, $68 p+$ annexes.

OMS, 2014. Surveillance de la qualité de l'eau de boisson. Genève, 1977, $143 p$.

Tandje J. F. R., 2009. Approvisionnement en eau potable dans la commune d'Adjarra: cas des arrondissements de Medejonou et d'Aglobe. Mémoire de maîtrise FLASH/UAC, $94 \mathrm{p}$.

Tchaba A. Y., 2012. Problématique de l'approvisionnement en eau potable dans la Commune de Comè Mémoire de maîtrise

Webographie FLASH/UAC, $77 \mathrm{p}$.

Http// : www.criteres.eau@mddefp.gouv.qc.ca

Http //:www.gouv.bj/communes/toff 